University of New Hampshire

University of New Hampshire Scholars' Repository

\title{
4-2014
}

\section{An image processing approach for determining of relative pose of unmanned underwater vehicles}

\author{
Yuri Rzhanov \\ University of New Hampshire, Durham, Yuri.Rzhanov@unh.edu \\ Firat Eren \\ University of New Hampshire, Durham, Firat.Eren@unh.edu \\ May-Win Thein \\ University of New Hampshire, Durham \\ Shachak Pe'eri \\ University of New Hampshire, Durham, shachak.peeri@unh.edu
}

Follow this and additional works at: https://scholars.unh.edu/ccom

\section{Recommended Citation}

Y. Rzhanov, Eren, F., Pe'eri, S., and Thein, M. - W., "An image processing approach for determining of relative pose of unmanned underwater vehicles", Oceans'14 MTS/IEEE. Taipei, Taiwan, 2014.

This Conference Proceeding is brought to you for free and open access by the Center for Coastal and Ocean Mapping at University of New Hampshire Scholars' Repository. It has been accepted for inclusion in Center for Coastal and Ocean Mapping by an authorized administrator of University of New Hampshire Scholars' Repository. For more information, please contact Scholarly.Communication@unh.edu. 


\section{An image processing approach for determining of relative pose of unmanned underwater vehicles}

\section{Yuri Rzhanov, Firat Eren, May-Win Thein and Shachak Pe'eri}

The use of a light source as a beacon is advantageous for the guidance and control of Unmanned Underwater Vehicles (UUVs). This approach allows a follower UUV to determine its relative pose (position and orientation) using low-cost commercial off the shelf (COTS) hardware (e.g., metal halide light sources). In order to design an effective detector unit for the follower UUV and predict its performance, a simulator program has been developed. The program simulates a light field using hardware and environmental parameters describing the light source, water properties, the detector unit geometry and electronic sensitivity. The simulator allows examination of different 3D detector array shapes of varying sizes (physical dimensions and number of detectors). It is convenient to present simulator output as an image, where each pixel represents the intensity logged by a corresponding detector. These image outputs are evaluated for the development of control algorithms for UUVs. Currently control algorithms assume that the water column is uniform with a systematic background noise. Considered control algorithms are able to provide guidance based on relative intensity values, where the light field samples on the detector array resembles a Gaussian beam pattern. However, disturbances in the medium (e.g., sediment plume) may cause non-uniform distribution of the scatterers that can distort the beam pattern. As a result, the control algorithms could misinterpret the acquired image and direct the follower UUV away from the guiding beam. The probability for such a situation increases with distance as the beam diverges. This paper suggests an alternative approach for the development of UUV control algorithms using calculations of various moments of the image (e.g. local Hessian estimations). This method allows the evaluation of the array performance with different array geometries and a varying number of detector elements.

The reference coordinate system assumes that the light source is at the origin, the X-axis is coincident with the center of the guiding beam, and the $Y$-axis is parallel to the rows of the detector array in the initial follower UUV position. The Z-axis is orthogonal to the XY plane, thus completing the orthogonal triad. The motion of the follower UUV can be described as a 5 degree of freedom (DOF) pose with respect to the light source: three translational motions (i.e., $X, Y$, and Z-displacements) and two rotations (yaw rotation about the $\mathrm{Y}$-axis and pitch rotation about the $\mathrm{Z}$-axis). Simulations have shown that it is not possible to decouple roll (rotation about the X-axis) from yaw or pitch because of the symmetry of the light beam. Instead, it is assumed that the follower has a stabilizer that maintains a fixed roll angle.

The location of the peak beam value, $\left(y_{0}, z_{0}\right)$ is determined with sub-pixel accuracy and is assumed to be within the array, where $y_{i}$ and $z_{j}$ are the row and line coordinates for a given detector in the array, respectively. The moments are defined as the weighted sum of the intensity values of the array detectors, $I_{i, j}$ with respect to $\left(y_{0}, z_{0}\right)$ :

$$
M_{p q}=\sum_{i, j}\left(y_{i}-y_{o}\right)^{p}\left(z_{j}-z_{o}\right)^{q} I_{i, j}
$$


It was demonstrated that it is sufficient to calculate only 5 moments for a 5 DOF pose, specifically, $M_{02}, M_{12}, M_{20}, M_{21}, M_{22}$. Specific functions of moments are chosen for each particular array geometry. Preliminary results show good performance for different array geometries. Relative pose determination is robust with respect to noise and number of detectors in the array. The performance evaluation of planar arrays shows that algorithm performance degrades with distance. The performance evaluation of curved arrays shows a relationship between the light field structure and the curvature of the array, in addition to distance. Image distortions that represent variable turbidity in water are also to be evaluated. 